\title{
The discrepancy between dynamical and theoretical mass in the triplet-system 2MASS J10364483+1521394 (Corrigendum)
}

\author{
Per Calissendorff ${ }^{1}$, Markus Janson ${ }^{1}$, Rainer Köhler ${ }^{2,3}$, Stephen Durkan ${ }^{4}$, Stefan Hippler ${ }^{5}$, Xiaolin Dai \\ Wolfgang Brandner ${ }^{5}$, Joshua Schlieder ${ }^{6}$, and Thomas Henning ${ }^{5}$ \\ ${ }^{1}$ Department of Astronomy, Stockholm University, Stockholm, Sweden \\ e-mail: per.calissendorff@astro.su.se \\ 2 Institut für Astro- und Teilchenphysik, Universität Innsbruck, Technikerstr. 25/8, 6020 Innsbruck, Austria \\ ${ }^{3}$ Department of Astrophysics, University of Vienna, Vienna, Austria \\ ${ }^{4}$ Astrophysics Research Centre, Queens University, Belfast, UK \\ ${ }^{5}$ Max Planck Institute for Astronomy, Heidelberg, Germany \\ ${ }^{6}$ NASA Goddard Space Flight Center, Greenbelt, MD 20771, USA
}

A\&A, 604, A82 (2017), https://doi.org/10.1051/0004-6361/201730725

An arithmetic error was made in the calculations of the optimal distance where the theoretical mass and dynamical mass would coincide. The updated estimation of the optimal distance is $17.2 \pm 0.2 \mathrm{pc}$, corresponding to both theoretical and dynamical masses of $\approx 0.15 M_{\odot}$ for the $\mathrm{B}$ and $\mathrm{C}$ components individually. These updated values only change the numbers in the discussion of the optimal distance and do not change the main results of the paper. 